\title{
Cot-741202.22
}

\section{ROLE OF ITINERANT $5 f$ STATES ON THE FERMI SURFACE \\ OF Th AND POSSIBLE IMPURITY LOCAL MOMENT FORMATION}

by

D. D. Koelling and A. J. Freeman

\begin{tabular}{|c|}
\hline 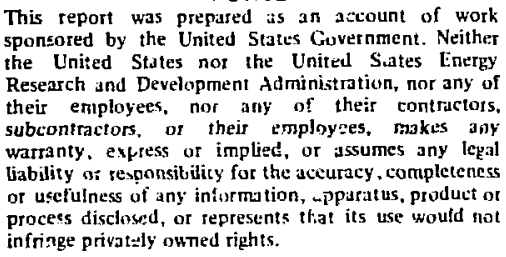 \\
\hline
\end{tabular}

Presented At:

20th Annual Conference on Magnetism and Magnetic Materials, San Francisco, California, Derember $3-6,1974$ 
ROLE OF ITINERAIT $5 F$ STATES ON THE FERMI SURFACE OF Th AND POSSIBLE IMPURITY LOCAL MOMENT FORMATION*

D. D. Koelling

Argonne National Laboratory, Argonne, I11inois 60439 and

A. J. Freeman

Physics Department, Northwestern University, Evanston, Illinois 60201 and Argonne National Laboratory, Argonne, Illinois 60439

ABSTRACT

The nature (itinerant vs. localized) of the $5 f$ electrons in the actinides determines their magnetic properties. Extensive optical and de Haas-van Alphen data which exist only for $T h$, the simplest actinide with no occupied $5 f$ states, allow detailed comparisons to be made between theory and experiment. The $5 f$ bands were found, by means of ab initio RAPW energy band calculations, to lie above the Fermi energy, to be itinerant in character and to significantly affect the reflectivity measurements of Veal, et al. We find that the $5 f$ bands when included in the RAPW calculations, give excellerit agreement with the de Haas-van Alphen results of Boyle and Gold, for all sections of the Lungs, Dumbel1 and Superegg Fermi surfaces, in contrast with calculations basel on artificialiy removing the $5 f$ resonance. Simple overlap charge density impurity scattering potential model calculations are used to discuss the possible occurrence of impurity local moment formation in Th based ailoys. $\sim$

\section{INTRODUCTION}

Theoretical studies of the actinide metals have shown that their magnetic and other properties are determined by the nature of their $5 f$ elections-itinerant for the light actinides ( $\mathrm{Th}-\mathrm{Pu}$ ) aull localized for the heavy actinides (Am and beyond). ' Unfortunately, the low temperature phases of the light actinides are structurally complex making extremely difficult both the band calculations and intarpretations of experimenta? data. Thorium, at the beginning of the series, with no occupied $5 f$ states, has the fcc structure as its low temperature phase and thus appears to be an ideal candidate for theoretical and experimental investigations. The availability of high purity single crystals has permitted accurate de Has-van Aiphen (dtvA) measurements to te made. Gupta aud Louciss ${ }^{2}$ artifically renoved the $5 f$ bands from their RAPW calculations, in order to overcoment the difficulties found by Keeton and Loucks ${ }^{3}$ in their ful! slater exchange $(c=1)$ calculations, and obtained reasonable agreement with the dHuA measurements. By contrast, Koelling and Freeman ${ }^{2}{ }^{4}$ argued 
that the $5 f$ electrons in the lighter actinides are sufficiently delocalized to significantly overlap neighboring atoms and to hybridize strongly with the $6 \mathrm{~d}$ and $7 \mathrm{~s}$ bands. As in the case of the lighter actinides, the $f$ electrons have small Coulomb correlation energy (relative to the now large effective band width) and hence must be considered itinerant; i.e., are to be included in and described by band calculations unlike the case of the $4 \mathrm{f}$ electrons in the rare earths.

Recently normal incidence reflectivity measurements on thorium by Veal, et al. ${ }^{5}$ showed the existence of band-like $f$ states $1-5 \mathrm{eV}$ above $\mathrm{E}_{\mathrm{F}}$. Because of the importance of this result ass providing strong confirming evidence for the prediction that the occupied $5 f$ states in the lighter actinide metals are itinerant, a highly precise determination of the FS of Th appears necessary as a severe test of the itinerant model.

We have found that the itinerant model (f states included) gives Fermi surface dimensions in excellent agreement with the dHvA measurements unlike the predictions of the localisci (f state removed) model. In addition, we have used the overlapping charge density impurity potential scattering model to calculate for the dilute impurity in Th problem some information about the rossible $f$ resonance states on the impurities and to discuss the possibility of impurity local moment formation.

\section{INFLUENCE OF UNOCCUPIED 5 S STATES ON THE FERMI SURFACE}

The relativistic energy band structure of fcc Th consists of $s$ and $d-1$ ike states as in any high $Z \mathrm{fcc}$ transition metal. ${ }^{1-4}$ For the itinerant model, there are in addition $f$ bands located in the range of 1 to $5 \mathrm{eV}$ above $E_{\mathrm{F}}$. In both models the Fermi Surface (FS) consists of three distinct pieces: (1) a hole surface at the center of the Brillouin zone (BZ) shaped like a rounded cube called "superegg"; (2) electron surfaces on symmetry lines $\Gamma K\langle 110\rangle$ shaped like pairs of "lungs"; and (3) the hole surfaces on the symmetry lines 「L<111> shaped like "dumbbells" with triangular ends. In comparing their model with experiment, ${ }^{6}$ Gupta and Loucks ${ }^{2}$ concluded that their model was "only in qualitative agreement with the experimental results". In many ways, the agreement obtained is rather remarkable but may be understood in terms of the potential insensitivity of the underlying transition-element band structure found by us from comparisons of the Fermi surfaces resulting from four different calculations: (1) the Gupta-Loucks (GL) calculation $\left(\mathrm{d}^{2} \mathrm{~s}^{2}, \alpha=1, \mathrm{f}\right.$ states removed); (2) the calculation for the $d^{2} s^{2}, \alpha=2 / 3$ potential with the $f$ states removed; (3) the calculation for the $d^{2} s^{2}, \alpha=2 / 3$ 
potential with the f states included; and (4) the calculation for the $d^{3} s^{1}, \alpha=2 / 3$ potential with $f$ states included.

The FS showed a very clear separation between the calculations with the $f$ states included and those with the $f$ states excluded. The two $F$ s resulting from the calculations (3) and (4) with the $f$ states included and with the configuration varied were close to identical. (This tends to give one confidence that these results will not differ greatly from a fully selfconsistent calculation.) The two FS for the $f$ states removed calculations give some index of the small but observable sensitivity to exchange approximation of the underlying transition-element band structure. Further, we find that the effect of changing $\alpha$ is to move the $f$ states relative to the remaining bands. Thus the parameter $\alpha$ main $1 y$ affects these other bands and their Fermi surface via the $f$ states and their hybridization with these bands.

Having (briefly) described the effects of selfconsistency (through configuration) and exchange approximation, we examine the effect on the FS of including or omitting the $f$ states. This effect was found to be larger than that due to change of configuration or exchange. The effect on the hole surfaces was to move volume fron the superegg to the dumbbell. The superegs shows somewhat more structure in addition to being smaller. The dumbbell increased in size primarily on the ball portion, the rod section toward $L$ remaining relatively unchanger. The electron surface (the lungs) is not centered on a hish-symmetry point but on a line and thus can change its position. The inclusion of the $f$ states moves this surface slightly in the direction of $\Gamma$. The tube which connects the two lobes across the $\Sigma$ line is reduced in size. The lobes themselves are taller and narrover. Qualitatively, these changes are all in the correct direction to improve the agrcement between theory and experiment.

However, in order to obtain a more quantitative comparison, one must actually perform the area integrals. The results of these calculations give dHvA areas in excellent agreement with the experiment tor all the experimental orbits observed by Boyle and $\operatorname{Gold}^{6}$ (provided their orbits $c$ and $d$ are interchanged). The effect of including the f-states was to reduce the largest discrepancies on the hole surfaces irom over $100 \%$ to less than $15 \%$. In addition, comparing the calculated values of $\mathrm{m}^{*}$ with the few experimental numbers give reasonable evhancement factors $(0.4$ to $0.7)$-again provided orbits $c$ and $d$ are interchanged. This excellent agreement with experiment adds strong confirmation to the itinerant nature of $5 f$ states in the lighter actinides. 
A number of striking effects are observed if dilute impurities are alloyed with Th metal. ${ }^{-10}$ As an ideal BCS superconductor with a transition temperature of $1.36^{\circ} \mathrm{K}$, Th has been used as a host for a number of alloy experiments which focus on the magnetic (pairbreaking) properties of the impurities since their dramatic effect on superconducting properties gives a sensitive test for magnetic moment formation. Thus the experimental results have been compared with Kaiser's ${ }^{11}$ theory for the effect of nonmagnetic resonant impurity states on the superconductivity of the iot metal and/or with the Abrikosov-Gorkov (AG) theory for the pairing breaking effect of a localized magnetic moment. It is found that: Ce behaves as a nonmagnetic impurity and fits the Kaiser theory; Gd, as a magnetic impurity fits the $A G$ theory; and $U$ is an intermediate case which is associated with localized spin fluctuations.

In order to understand the results of these experiments, we have examined the overlapping charge density model for a single inpurity in a Th host. Within this model (which is not necessarily self-consistent), we can then hope to see the nature of any f-resonances which might form, and relate them to the possibility of local moment formation. Thus we superyose (overlap) the impurity charge density and the host atom charge densities, construct a mufifin tin (MT) potential and solve the radial Dirac equa'sion to determine the (logarithmic derivatives and the) scattered-wave phase shifts. To define the quantities of interest we note that this procedure applied to a pure metal (and excluding hybridization) would yield $\varepsilon_{\text {min }}^{j}$ and $\varepsilon_{\text {tnax }}^{j}$ (relative to $E_{F}$ ) for which the maximum of the wave function and its zero (which would be at infinity for the free atom) occur at the MT radius. These would be upper and lower bounds for the states with a given $j$-character and so give some idea of the energy range over which $f$ resonances will have an appreciable effect. The other two parameter $\epsilon_{\mathbf{r}}^{\mathbf{j}}$ and $\Gamma^{j}$ are obtained from the expansion in energy

$$
\cot \delta_{j}=\frac{\varepsilon_{r}^{j}-\varepsilon}{\Gamma^{j}}+\ldots
$$

of the cotangent of the phase shift near the energy for which it is zero. This expansion has been used extensively in deriving combined basis representations from the Green's function formalism; ${ }^{22} \varepsilon_{\vec{r}}^{j}$ is the resonant energy (center of the band for a collection of such potentials) and $\Gamma^{j}$ is the Born transition 
probability. [To establish the scale of $\mathrm{T}$, we note that $J=0.044 \mathrm{ky}$ for the d-states in bec Fe.] Clearly it will be the difference of the impurity values from the Th values which are significant since if the impurity scattered like Th it would have no effect on the electron states.

Our results for $\mathrm{Ce}, \mathrm{Gd}$ and $\mathrm{U}$ are given in Table $\mathrm{I}$, for $j=5 / 2$ and $7 / 2$ of each impurity. The results for Gd show quite clearly that since the transition probability $\Gamma$ is small and the resonance lies well below the $E_{F}$ one will obtain a local moment on the impurity site. (Because of the large Coulomb correlation energy, $\mathrm{U}_{\mathrm{eff}}$, an occupied $\mathrm{f}^{\mathrm{B}}$ configuration would move far above the Fermi energy; thus these one-electron energies must be used with care when the states are beginning to localize.) The results show quite clearly that $C e$ will not support any $f$ states but that one will get considerable scattering. Since the "clear-cut" cases of $\mathrm{Gd}$ and $\mathrm{Ce}$ are seen to fit well into our model, we now consider the case of $U$.

It is interesting to note that $U\left(f^{11} d^{2} s^{1}\right)$ yields nearly the same scattering parameters as Th. Thus, if $U$ were to remain in this configuration, it would have very little effect on the Th host. However, as this would require the occupied f-states to remain in their energy states vell above the Fermi energy, this cannot be the ground state of the system. If, instead, we look at $U\left(\mathrm{f}^{2} \mathrm{~d}^{3} \mathrm{~s}^{1}\right)$, the $\mathrm{f}$ levels are lowered by $0.16 \mathrm{Ry}$ (which gives an estimate of the Covlomb correlation energy) and can be occupied. Here we notice that the widths $\Delta=\left(\varepsilon_{\max }^{j}-\varepsilon_{\min }^{j}\right)$ anc/or transitior. probabilities $\Gamma^{j}$ are reduced to $40 \%$ of their $U\left(\varepsilon^{3} \mathrm{~d}^{2} \mathrm{~s}^{1}\right)$ values because the atomic $f$ states have contracterl by roughly $10 \%$. Hence, for the case of $U$ impurities, the f states are located at $\mathrm{E}_{\mathrm{F}}$ as required by the specific heat data $\mathrm{a}^{10}$ and self-consistency requirements, aise much more localized than with the trivalent configuration, and strongly correlated (Ueff $\approx \Delta$ ).

Table I. f-state parameters (in Ry) for impurities in Th

\begin{tabular}{|c|c|c|c|c|c|c|c|c|}
\hline Impurity & $\varepsilon_{\min }^{5 / 2}$ & $\varepsilon_{\max }^{5 / 2}$ & $\varepsilon_{r}^{5 / 2}$ & $\Gamma^{5 / 2}$ & $\varepsilon_{\min }^{7 / 2}$ & $\varepsilon_{\text {Eax }}^{7 / 2}$ & $\varepsilon^{7 / 2}$ & $\Gamma^{7 / 2}$ \\
\hline $\operatorname{Th}\left(\mathrm{d}^{3} \mathrm{~s}^{1}\right)$ & 0.06 & 0.30 & 0.19 & 0.031 & 0.09 & 0.37 & 0.24 & 0.038 \\
\hline $\operatorname{Gd}\left(\varepsilon^{7} \dot{d}^{2} \dot{s}^{3}\right)$ & -0.13 & -0.09 & -0.10 & 0.001 & -0.07 & -0.04 & -1.06 & 0.002 \\
\hline $\operatorname{Ce}\left(f^{1} d^{2} s^{1}\right)$ & 0.11 & 0.20 & 0.15 & 0.009 & 0.12 & $0.2 ?$ & 038 & 0.012 \\
\hline $\operatorname{Ce}\left(\mathrm{d}^{3} \mathrm{~s}^{1}\right)$ & 0.10 & 0.20 & 0.15 & 0.011 & 0.12 & 0.22 & 0.1. & 0.012 \\
\hline$U\left(f^{3} d^{2} s^{1}\right)$ & 0.10 & 0.30 & 0.20 & 0.025 & 0.15 & 0.36 & 0.26 & 0.032 \\
\hline$U\left(F^{2} d^{3} s^{2}\right)$ & .0 .06 & 0.09 & 0.02 & 0.010 & -0.01 & 0.16 & $0.0 i i$ & 0.015 \\
\hline
\end{tabular}


* Supported by the AEC, the NSF and the AFOSR.

1. See the review article by A. J. Freeman and D. D. Koelling, in the Actinides: Electronic Structure and Properties, A. J. Freenan and J. B. Darby, Jr., Eds. (Academic Press, N.Y., 1974), Vol. I, P. 51.

2. R. P. Gupta and T. L. Loucks, Phys. Rev. Letters 22,458 (1969).

3. S. C. Keeton and T. L. Louscks, Phys. Rev. 146, 429 (1966).

4. D. D. Koelling and A. J. Freeman, Solid State Commun Conmun. 9 , 1369 (1971).

5. B. W. Veal, D. D. Koelling, and A. J. Freeman, Phys. Rev. Letters 30, 1061 (1973).

6. D. J. Boyle and A. V. Gold, Phys. Rev. Letters 22, 461 (1969).

7. W. R. Decker and D. K. Finnemore, Phys. Rev. 172, 430 (1968).

8. J. G. Huber and M. B. Maple, J. Low Temp. Phys. 3, 537 (1970).

9. M. B. Maple, J. G. Huber, B. R. Coles, and A. C. Lawson, J. Low Temp. Phys. 3, 137 (1970); H. L. Watson, D. T. Peterson, and D. K. Finnemore, Low Temperature Physiss-LT 13, K. D. Timmerhaus, W. J. O'Sullivan, and E. F. Hammel, Eds. (Plenum Press, N.Y., 1974), Vol. II, p. 590.

10. C. A. Luengo, J. M. Cotignola, J. Sereni, A. R. Sweedler, and M. B. Maple, Low Temperature Physics-LT 13, K. D. Timmerhause, H. J. 0'Sullivan, and E. F. Hamme1, Eds. (Academic Press, N.Y., 1974), Vol. II, p. 585.

11. A. B. Kaiser, J. Phys. C 3 , 409 (1970).

12. D. G. Pettifor, J. Phys. C 3,367 (1970). 